\title{
Dietary determinants of iron intake in women of child-bearing age (WCBA) (18-50y) in Ireland
}

\author{
Amy Fahy ${ }^{1,2}$, Laura Kehoe ${ }^{1}$, Janette Walton ${ }^{2}$, Breige A. McNulty ${ }^{3}$, Anne P. Nugent ${ }^{4}$ and \\ Albert Flynn ${ }^{\mathrm{T}}$ \\ ${ }^{1}$ School of Food and Nutritional Sciences, University College Cork, Ireland, Cork, Ireland, \\ ${ }^{2}$ Dept. Biological Sciences, Cork Institute of Technology, Ireland, Cork, Ireland, \\ ${ }^{3}$ UCD Institute of Food and Health, University College Dublin, Ireland, Dublin, Ireland and \\ ${ }^{4}$ Institute for Global Food Security, Queens University Belfast, Belfast, United Kingdom
}

\section{Abstract}

Dietary iron requirements are higher among women of child-bearing age (WCBA) to replenish blood loss during menses, to prevent iron deficiency anaemia and to support a healthy foetus during pregnancy. Low intakes of iron have previously been reported among WCBA in Ireland and across Europe and data from European countries have shown that there is evidence of anaemia and low iron stores in this population group. The aim of this study was to investigate the dietary patterns influencing iron intakes in WCBA (18-50 years) in Ireland.

Analyses were based on the National Adult Nutrition Survey (NANS) (2008-2010) (www.iuna.net). A 4 day semi-weighed food record was used to collect food and beverage intake data (including nutritional supplement use) from a nationally representative sample of 1500 adults (487 women (18-50y)). Nutrient intakes were estimated using WISP ${ }^{\odot}$ based on UK and Irish food composition data. On the basis of mean daily intake of iron, WCBA were divided into three intake groups: low, medium and high. The food groups that accounted for the greatest proportion of the difference between the high (top third) and low (bottom third) of iron intakes were identified.

The difference in iron intakes between the high and low intake groups $(25 \mathrm{v} 6.7 \mathrm{mg} / \mathrm{d})$ was $18.3 \mathrm{mg} / \mathrm{d}$. Nutritional supplements and ready-to-eat breakfast cereals (RTEBC) (commonly fortified with iron) accounted for 58 and 20\% of the difference in intakes between the high and low intake group, respectively. The contribution of nutritional supplements to the difference in iron intakes can be explained by the proportion of users in the high vs low intake group $(27 \%$ vs $<1 \%)$. The contribution of RTEBC to the difference in iron intakes can be explained by both a higher proportion of those in the high vs low intake group consuming RTEBC and those in the high intake group having a higher mean daily intake of RTEBC (78\% vs 36\%; 32g/d vs $7 \mathrm{~g} / \mathrm{d}$ ).

Most of the difference in iron intake between high and low consumers is attributable to nutritional supplement use and the patterns of consumption of fortified RTEBC. These findings will aid in the development of strategies to improve iron intakes in WCBA in Ireland.

\section{Conflict of Interest}

There is no conflict of interest. 